\title{
Endoproteinase Lys-C Protein Cleavage Reagent
}

National Cancer Institute

\section{Source}

National Cancer Institute. Endoproteinase Lys-C Protein Cleavage Reagent. NCI

Thesaurus. Code C161886.

A protein cleavage reagent comprised of the bacterial endoproteinase Lys-C that cleaves

proteins into peptides at the C-terminal side of lysine residues unless that residue is followed by proline. 